\title{
THE HUREWICZ HOMOMORPHISM AND FINITE HOMOTOPY INVARIANTS
}

\author{
BY \\ M. ARKOWITZ AND C. R. CURJEL(1)
}

1. Introduction. It has been proved by Berstein [2] that the cokernel of the Hurewicz homomorphism $h_{n}: \pi_{n}(X) \rightarrow H_{n}(X)$ is a finite group if the space $X$ is of Lusternik-Schnirelmann category $<2$ modulo the class $\mathscr{F}$ of finite abelian groups. On the other hand, if $X$ is an $H$-space mod $\mathscr{F}$ then it follows from results of CartanSerre [6] that the kernel of $h_{n}$ is finite. In this paper we establish converses of these results for a wide class of spaces by studying in general conditions under which the cokernel or the kernel of the Hurewicz homomorphism $h_{n}: \pi_{n}(X) \rightarrow H_{n}(X)$ is a finite group.

We first discuss the case where $h_{n}$ is an $\mathscr{F}$-epimorphism, i.e., the cokernel of $h_{n}$ is in $\mathscr{F}$. Consider a homology decomposition, the dual of a Postnikov decomposition, of $X$ and denote by $k_{n}^{\prime}$ its $n$th $k^{\prime}$-invariant [10]. In Proposition 2.1 we prove that $h_{n}$ is an $\mathscr{F}$-epimorphism if and only if $k_{n}^{\prime}$ is of finite order. It is also seen that this latter condition holds if and only if $X$ is homologically equivalent $\bmod \mathscr{F}$ in dimension $n$ to a wedge of $n$-spheres. Next we turn our attention to those spaces for which the Hurewicz homomorphism is an $\mathscr{F}$-epimorphism in all dimensions. Let us call a map a weak $\mathscr{F}$-equivalence if it induces $\mathscr{F}$-isomorphisms on integral homology. The main result of $\$ 2$ (Theorem 2.5 ) then asserts that, for a finite polyhedron $X$, the following five conditions are equivalent:

(1) The homomorphism $h_{n}: \pi_{n}(X) \rightarrow H_{n}(X)$ is an $\mathscr{F}$-epimorphism for all $n$.

(2) All $k^{\prime}$-invariants of $X$ are of finite order.

(3) There exists a weak $\mathscr{F}$-equivalence from a wedge of spheres into $X$.

(4) There exists a weak $\mathscr{F}$-equivalence from $X$ into a wedge of spheres.

(5) The Lusternik-Schnirelmann category $\bmod \mathscr{F}$ of $X$ is less than 2.

The equivalence of statements (2) and (5) is a generalization and converse of a result due to Curjel [8]. The equivalence $(3) \Leftrightarrow(4)$ provides a partial answer to the following question raised by Serre for an arbitrary class of abelian groups $[16$, p.290]: Given a weak $\mathscr{F}$-equivalence $f: Y \rightarrow X$, does there exist a weak $\mathscr{F}$-equivalence $g: X \rightarrow Y$ in the opposite direction? In Example 2.7 we present a space $X$ and a weak $\mathscr{F}$-equivalence $f: S^{n} \rightarrow X$ and prove that there is no map $g: X \rightarrow S^{n}$ which is a weak $\mathscr{F}$-equivalence. This example is of twofold interest.

Received by the editors January 31, 1963.

(1) During the time that this work was done, the first author was partially supported by the Air Force Office of Scientific Research and the second author by NSF Grant G-15984. 
First of all, it shows that Theorem 2.5 does not hold without some restrictions on $X$. Secondly, it gives a negative answer, at least for the class $\mathscr{F}$, to Serre's question.

In $\S 4$ we study conditions under which the kernel of $h_{n}: \pi_{n}(X) \rightarrow H_{n}(X)$ is in $\mathscr{F}$. It is shown in Proposition 4.1 that $h_{n}$ is an $\mathscr{F}$-monomorphism if and only if, for some Postnikov decomposition $\left\{X^{n}\right\}$ of $X$, the $k$-invariant in $H^{n+1}\left(X^{n-1} ; \pi_{n}(X)\right)$ is of finite order. This occurs if and only if there is a map of $X$ into a product of $K(Z, n)$ 's which induces an $\mathscr{F}$-isomorphism on the $n$th homotopy groups. The main result of $\S 4$ (Theorem 4.5 ) deals with spaces for which $h_{n}$ is an $\mathscr{F}$-monomorphism for all $n$. This result, as its dual (Theorem 2.5), asserts, under certain assumptions, the equivalence of five properties of $X$. Three of them are (1) $h_{n}$ is an $\mathscr{F}$-monomorphism for all $n$. (2) All $k$-invariants of $X$ are of finite order. (3) $X$ is an $H$-space mod $\mathscr{F}$. The other two deal with weak $\mathscr{F}$-equivalences from and into a product of $K(Z, n)$ 's. The implication $(1) \Rightarrow(3)$ is a converse to the result of Cartan and Serre mentioned above. Furthermore, the implication $(2) \Rightarrow(3)$ is a converse to Thom's theorem on the $k$-invariants of an $H$-space $\bmod \mathscr{F}$ [18]. We note that Example 2.7 is of interest also in connection with Theorem 4.5.

By combining the results on the kernel and cokernel of $h_{n}$, we characterize in Theorem 6.4 the spaces for which the Hurewicz homomorphism is an $\mathscr{F}$-isomorphism in all dimensions. These spaces turn out to be rational homology spheres of odd dimension.

The paper consists of six sections. The cokernel of $h_{n}$ is considered in $\S 2$, and $\S 3$ is devoted to the proof of Theorem 2.5. This proof depends on a divisibility property of the homotopy groups of a double suspension (Proposition 3.1) which is based on Barratt's discussion of the distributive law [1]. The kernel of $h_{n}$ is studied in $\$ 4$, and the proof of Theorem 4.5 occupies $\S 5$. The main lemma which is needed for this proof utilizes some results on cohomology operations. In $\$ 6$ conditions under which $h_{n}$ is an $\mathscr{F}$-isomorphism are investigated. For the remainder of $\S 1$ we present our notation and recall some definitions and facts.

We consider only 1-conneced topological spaces with a base point. All spaces are assumed to have the homotopy type of a polyhedron (that is, a CW-complex) and to have finitely generated integral homology groups in each dimension. All maps and homotopies are to keep the base point fixed. For notational convenience we do not distinguish between a map $f: X \rightarrow Y$ and its homotopy class in the set $\pi(X, Y)$. For an abelian grup $G$, the induced homomorphism on homology groups (respectively, cohomology groups) is written $f_{*}: H_{n}(X ; G) \rightarrow H_{n}(Y ; G)$ (respectively, $\left.f^{*}: H^{n}(Y ; G) \rightarrow H^{n}(X ; G)\right)$, and the induced homomorphism on homotopy groups is written $f_{*}: \pi_{n}(X) \rightarrow \pi_{n}(Y)$. We denote by $K^{\prime}(G, n)$ a Moore space of type $(G, n)$ (i.e., a space with a single non-vanishing homology group $G$ in dimension $n$ ) and by $K(G, n)$ an Eilenberg-MacLane space of type $(G, n)$. The $n$th homotopy group of $X$ with coefficients in $G$ is defined as $\pi\left(K^{\prime}(G, n), X\right)$ and written $\pi_{n}(G ; X)$. We denote by $\Sigma$ the reduced suspension functor and also the suspension homo- 
morphism $\Sigma: \pi_{n}(X) \rightarrow \pi_{n+1}(\Sigma X)$. There is always a comultiplication map $\Sigma X \rightarrow \Sigma X \vee \Sigma X$ which determines a group structure in $\pi(\Sigma X, Y)$. We let $\Omega$ stand for the loop space functor and also for the corresponding homomorphism of homology or cohomology groups (e.g., $\Omega: H_{n-1}(\Omega X ; G) \rightarrow H_{n}(X ; G)$ ).

As already mentioned, $\mathscr{F}$ denotes the class of finite abelian groups, and a map $f: X \rightarrow Y$ is called a weak $\mathscr{F}$-equivalence if $f_{*}: H_{n}(X) \rightarrow H_{n}(Y)$ is an $\mathscr{F}$-isomorphism for all $n$. It follows from a result of Serre [16, Théorème 3] that $f: X \rightarrow Y$ is a weak $\mathscr{F}$-equivalence if and only if $f_{\#}: \pi_{n}(X) \rightarrow \pi_{n}(Y)$ is an $\mathscr{F}$-isomorphism for all $n$.

The following notation shall be used consistently throughout this paper: (1) $h_{n}, h_{n}^{\prime}$ and $h_{n}^{\prime \prime}$ for the Hurewicz homomorphism in dimension $n$, (2) $Q$ for the additive group of rational numbers, (3) Id for the identity map of a space, (4) $C X \cup_{f} Y$ for the space obtained by attaching a cone over $X$ to $Y$ by means of $f: X \rightarrow Y$.

2. The cokernel of the Hurewicz homomorphism. We first recall the notion of a homology decomposition $\left\{X_{n} ; k_{n}^{\prime}\right\}$ of a space $X[10]$. This consists of:

(1) A sequence of polyhedra $X_{n}$ such that $H_{i}\left(X_{n}\right)=0, i>n$, together with maps $g_{n}: X_{n} \rightarrow X$ which induces homology isomorphisms in dimensions $\leqq n(n=2,3, \cdots)$.

(2) A sequence of elements $k_{n}^{\prime} \in \pi_{n-1}\left(H_{n}(X) ; X_{n-1}\right)$ such that

$$
X_{n}=C K^{\prime}\left(H_{n}(X), n-1\right) \cup_{k_{n}^{\prime}} X_{n-1} .
$$

The polyhedron $X_{n}$ is called the $n t h$ homology section and the element $k_{n}^{\prime}$ the $n t h k^{\prime}$-invariant of $\left\{X_{n} ; k_{n}^{\prime}\right\}$. We denote the inclusion by $j_{n}: X_{n-1} \rightarrow X_{n}$ and the projection by $p_{n}: X_{n} \rightarrow X_{n} / X_{n-1}=K^{\prime}\left(H_{n}(X), n\right)$. The maps $g_{n}$ and $j_{n}$ satisfy the relation

$$
g_{n} j_{n}=g_{n-1}: X_{n-1} \rightarrow X
$$

Proposition 2.1. The following three assertions are equivalent:

(1) The Hurewicz homomorphism $h_{n}: \pi_{n}(X) \rightarrow H_{n}(X)$ is an $\mathscr{F}_{\text {-epimorphism }}$ (i.e., the cokernel of $h_{n}$ is in $\mathscr{F}$ ).

(2) For any homology decomposition $\left\{X_{n} ; k_{n}^{\prime}\right\}$ of $X$, the nth $k^{\prime}$-invariant $k_{n}^{\prime}$ is an element of finite order in $\pi_{n-1}\left(H_{n}(X) ; X_{n-1}\right)$.

(3) There exists a map $\lambda$ from a wedge of $n$-spheres into $X, \lambda: S^{n} \vee \cdots \vee S^{n} \rightarrow X$, such that $\lambda_{*}: H_{n}\left(S^{n} \vee \cdots \vee S^{n}\right) \rightarrow H_{n}(X)$ is an $\mathscr{F}$-isomorphism.

Proof. First we show that $(1) \Leftrightarrow(2)$. Consider the commutative diagram

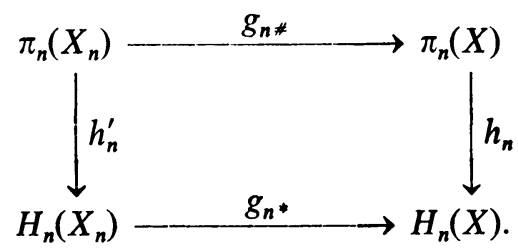


Since $g_{n}$ induces homology isomorphisms in dimensions $\leqq n$, the map $g_{n *}$ is an epimorphism [12, pp. 167-168]. Thus $h_{n}$ is an $\mathscr{F}$-epimorphism if and only if $h_{n}^{\prime}$ is an $\mathscr{F}$-epimorphism.

It is easily seen (e.g., [3, Theorem II]) that the sequence $X_{n-1} \rightarrow X_{n} \rightarrow \Sigma K^{\prime}$, with $K^{\prime}=K^{\prime}\left(H_{n}(X), n-1\right)$, gives rise to a commutative diagram with exact row

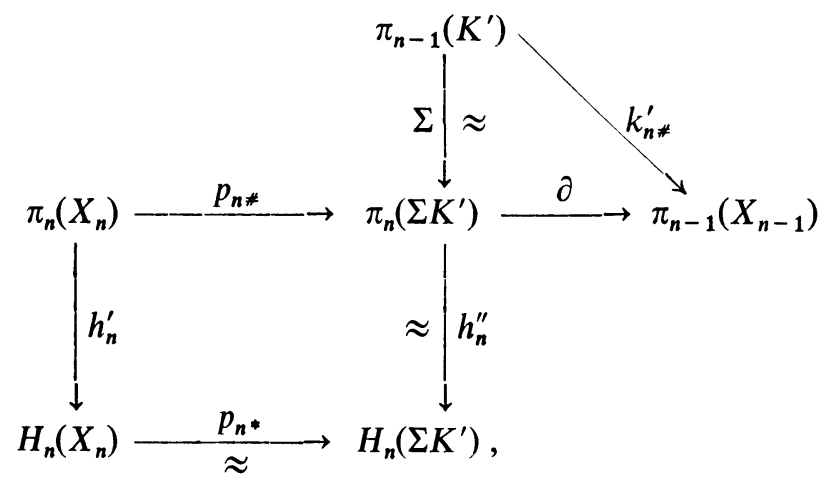

where $\partial$ is a boundary homomorphism and $\Sigma$ the suspension homomorphism. It follows from the properties of a homology decomposition that $p_{n^{*}}$ is an isomorphism. Furthermore $h_{n}^{\prime \prime}$ is an isomorphism by the Hurewicz theorem, and so

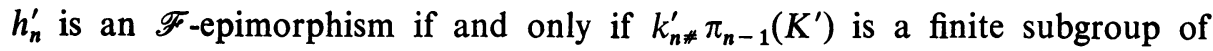
$\pi_{n-1}\left(X_{n-1}\right)$. Since $K^{\prime}=S^{n-1} \vee \cdots \vee S^{n-1} \vee K^{\prime}(T, n-1)$, where $T$ is a finite abelian group, the last assertion holds if and only if $k_{n}^{\prime} \mid S^{n-1} \vee \cdots \vee S^{n-1}$ is of finite order. But this is true precisely when $k_{n}^{\prime}$ is of finite order. Consequently we have shown that $h_{n}: \pi_{n}(X) \rightarrow H_{n}(X)$ is an $\mathscr{F}$-epimorphism if and only if $k_{n}^{\prime}$ is of finite order. This proves $(1) \Leftrightarrow(2)$.

Next we show that $(3) \Rightarrow(1)$. Since $\lambda_{*}: H_{n}\left(S^{n} \vee \cdots \vee S^{n}\right) \rightarrow H_{n}(X)$ is an $\mathscr{F}$ isomorphism, we see that $h_{n}: \pi_{n}(X) \rightarrow H_{n}(X)$ is an $\mathscr{F}$-epimorphism if $h_{n}^{\prime}: \pi_{n}\left(S^{n} \vee \cdots \vee S^{n}\right) \rightarrow H_{n}\left(S^{n} \vee \cdots \vee S\right)$ is an $\mathscr{F}$-epimorphism. The result now follows because $h_{n}^{\prime}$ is an isomorphism.

To prove $(1) \Rightarrow(3)$ we choose a basis $x_{1}, \cdots, x_{r}$ of the free part of $H_{n}(X)$. Since $h_{n}$ is an $\mathscr{F}$-epimorphism, there exist integers $N_{i}>0$ and elements $\lambda_{i} \in \pi_{n}(X)$ such that

$$
h_{n}\left(\lambda_{i}\right)=N_{i} x_{i}, \quad i=1, \cdots, r .
$$

The $\lambda_{i}$ determine a map $\lambda: S^{n} \vee \cdots \vee S^{n} \rightarrow X$ with the desired property.

REMARK 2.2. If $X$ is an $(r-1)$-connected space, then it is not difficult to verify that $k_{n}^{\prime}$ is of finite order for $n \leqq 2 r-1$ (e.g., apply Thom's lemma [7, Proposition 3.3] to $\left.k_{n}^{\prime} \mid S^{n-1} \vee \cdots \vee S^{n-1}\right)$. By combining this with Proposition 2.1 we retrieve the known fact that $h_{n}: \pi_{n}(X) \rightarrow H_{n}(X)$ is an $\mathscr{F}$-epimorphism for $n \leqq 2 r-1$.

Remark 2.3. Proposition 2.1 shows that the property that $k_{n}^{\prime}$ has finite order is an invariant of the homotopy type of $X$. This is in contrast to the fact that the 
homology decomposition is not an invariant of homotopy type. Indeed, Brown and Copeland [5, pp. 316-317] have exhibited two homotopically equivalent spaces $X$ and $Y$ with homology decompositions $\left\{X_{n} ; k_{n}^{\prime}\right\}$ and $\left\{Y_{n} ; l_{n}^{\prime}\right\}$ such that for some $m$ : (i) $X_{m}$ and $Y_{m}$ do not have the same homotopy type, (ii) $k_{m}^{\prime}=0$ and $l_{m}^{\prime} \neq 0$.

In order to state Theorem 2.5 we need the following definitions.

Definitions 2.4. (a)We say that a space $X$ has finite $k^{\prime}$-type if in some homology decomposition $\left\{X_{n} ; k_{n}^{\prime}\right\}$ of $X$, each $k_{n}^{\prime}$ is of finite order. (In view of Remark 2.3 this is an invariant notion.)

(b) We say that $\mathscr{F}$-cat $X<2$ if there exists a map $\mu: X \rightarrow X \vee X$ such that the composition of $\mu$ with each of the two projections of $X \vee X$ onto $X$ is a weak $\mathscr{F}$-equivalence.

The notion of $\mathscr{F}$-cat $X$ is due to Berstein [2]. We have normalized it by subtracting 1 .

THEOREM 2.5. Consider the following assertions:

(1) The Hurewicz homomorphism $h_{n}: \pi_{n}(X) \rightarrow H_{n}(X)$ is an $\mathscr{F}$-epimorphism for all $n$.

(2) The space $X$ has finite $k^{\prime}$-type.

(3) There exists a weak $\mathscr{F}$-equivalence

$$
\phi: S^{n_{1}} \wedge \cdots \wedge S^{n_{k}} \rightarrow X\left(n_{1} \leqq \cdots \leqq n_{k}\right) .
$$

(4) There exists a weak $\mathscr{F}$-equivalence $\psi: X \rightarrow S^{n_{1}} \vee \cdots \vee S^{n_{k}}$.

(5) $\mathscr{F}$-cat $X<2$.

Then the following conclusions hold:

(i) $(1) \Leftrightarrow(2)$.

(ii) If $H_{i}(X)$ is a finite group for all $i$ sufficiently large, then

$$
(1) \Leftrightarrow(2) \Leftrightarrow(3) \text {. }
$$

(iii) If $H_{i}(X)=0$ for all $i$ sufficiently large(e.g., $X$ is a finite polyhedron), then

$$
(1) \Leftrightarrow(2) \Leftrightarrow(3) \Leftrightarrow(4) \Leftrightarrow(5) \text {. }
$$

The proof of Theorem 2.5 occupies $\S 3$. We note that the equivalence $(2) \Leftrightarrow(5)$ constitutes a generalization and converse of a result of Curjel [8, Theorem I]. The implication $(5) \Rightarrow(1)$ is due to Berstein [2] and holds without the hypothesis of (iii). Some of the other implications also hold under weaker conditions. However, it is impossible to suppress all finiteness assumptions in (iii), as will be seen in Example 2.7.

COROLlary 2.6. Let $f: X \rightarrow Y$ be a weak $\mathscr{F}$-equivalence, where $H_{i}(X)$ and $H_{i}(Y)$ vanish for all i sufficiently large.

(a) Then $\mathscr{F}$-cat $X<2$ if and only if $\mathscr{F}$-cat $Y<2$. 
(b) If either $X$ or $Y$ has finite $k^{\prime}$-type (or, equivalently, is of $\mathscr{F}$-cat $<2$ ), then there exists a weak $\mathscr{F}$-equivalence $g: Y \rightarrow X$ in the opposite direction (i.e., the weak $\mathscr{F}$-equivalence can be "reversed").

The proof of (a) is a consequence of the equivalence $(1) \Leftrightarrow(5)$. For the proof of (b) observe that, if one of the two spaces has finite $k^{\prime}$-type, then so does the other. Hence the wedges of spheres associated with $X$ and with $Y$ are identical. Call this wedge $L$ and let $\phi: L \rightarrow X, \psi: Y \rightarrow L$ be the corresponding weak $\mathscr{F}$-equivalences. Then $g=\phi \psi: Y \rightarrow X$ is a weak $\mathscr{F}$-equivalence in the desired direction.

Corollary 2.6 (b) demonstrates that under certain conditions a weak $\mathscr{F}$-equivalence can be reversed. However, the following result shows that this is not true in general.

EXAMPLE 2.7. There is a weak $\mathscr{F}$-equivalence $f: S^{n} \rightarrow K(Z, n), n$ odd $\geqq 3$, but there is no weak $\mathscr{F}$-equivalence $g: K(Z, n) \rightarrow S^{n}$.

Proof. Any nonzero element $f$ in $\pi_{n}(K(Z, n))$ is a weak $\mathscr{F}$-equivalence. Now assume that there exists a weak $\mathscr{F}$-equivalence $g: K(Z, n) \rightarrow S^{n}$. Then $g f: S^{n} \rightarrow S^{n}$ is a weak $\mathscr{F}$-equivalence and therefore of degree $N \neq 0$. By a result of Serre $\left[16\right.$, Proposition 1, p. 278] the homomorphism $(g f)_{*}: \pi_{i}\left(S^{n}\right) \rightarrow \pi_{i}\left(S^{n}\right)$ is a $\mathscr{C}$-isomorphism, where $\mathscr{C}$ is the class of finite groups whose order divides a power of $N$. However, $(g f)_{*}=0$ for $i>n$, since $(g f)_{*}$ factors through $\pi_{i}(K(Z, n))$. Thus for all $i>n$ the order of $\pi_{i}\left(S^{n}\right)$ divides a power of $N$. On the other hand, it is known that for each prime $p$ there are infinitely many $j$ such that $p$ divides the order of $\pi_{j}\left(S^{n}\right)$ (see [13, Theorem 2]). This means that every prime divides $N$, which is impossible. Hence there is no weak $\mathscr{F}$-equivalence $g: K(Z, n) \rightarrow S^{n}$.

This example also shows that the implication $(3) \Rightarrow(4)$ of Theorem 2.5 does not hold if $H_{i}(X)$ is merely a finite group for all $i$ sufficiently large.

3. Proof of Theorem 2.5. Parts (i) and (ii) of Theorem 2.5 follow directly from Proposition 2.1.

Now we show that (3) and (4) imply (5). Let $\mu: L \rightarrow L \vee L$ be any comultiplication of $L=S^{n_{1}} \vee \cdots \vee S^{n_{k}}$. Define $\mu^{\prime}$ by $\mu^{\prime}=(\phi \vee \phi) \mu \psi$ :

$$
X \stackrel{\psi}{\longrightarrow} L \stackrel{\mu}{\longrightarrow} L \vee L \stackrel{\phi \vee \phi}{\longrightarrow} X \vee X .
$$

Thus it is seen that $\mathscr{F}$-cat $X<2$.

To prove (1) from (4) we replace $X$ by $L=S^{n_{1}} \vee \cdots \vee S^{n_{k}}$. Let $p$ be the projection of $L$ onto the $n$-spheres of $L, p: L \rightarrow S^{n} \vee \cdots \vee S^{n}$. The following diagram establishes (1):

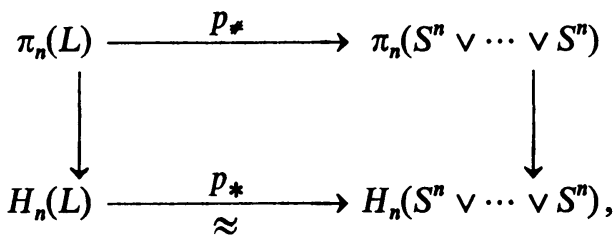


since $p_{\# \text { \# }}$ is an epimorphism.

As already noted the implication $(5) \Rightarrow(1)$ has been proved by Berstein $[2, \S 3]$. To complete the proof of Theorem 2.5 it only remains to show that (3) implies (4) under the assumption $H_{i}(X)=0$ for large $i$. The argument depends on the following proposition which may be of independent interest.

Proposition 3.1. For any space A (not necessarily 1-connected), any finitely generated group $G$ and integers $q>0, s>0$, there exists an integer $N>0$ such that

$$
\mathbf{N}_{\#} \pi_{q}\left(G ; \Sigma^{2} A\right) \subset s \cdot \pi_{q}\left(G ; \Sigma^{2} A\right),
$$

where $\mathbf{N}: \Sigma^{2} A \rightarrow \Sigma^{2} A$ represents $N$ times the identity map in $\pi\left(\Sigma^{2} A, \Sigma^{2} A\right)$.

Proof. Since $\mathbf{N}_{*} x=(\mathrm{Id}+\cdots+\mathrm{Id}) x$ is in general different from $N x$, we turn to a result of Barratt which is based on the Milnor-Hilton theorem. We restate Barratt's Lemma 6.8 of [1] with the following modifications:

(1) We use homotopy groups with coefficients $G$ in place of ordinary homotopy groups (see the remark on p. 130 of [1]).

(2) For Barratt's $\bar{u}_{\tau}^{\circ} H_{\tau}$ we write $\bar{H}_{\tau}$. Then for any $x \in \pi_{q}\left(G ; \Sigma^{2} A\right)$,

$$
\mathbf{N}_{*} x=N x+\Sigma_{\tau} \sigma_{\tau}(N) \bar{H}_{\tau}(x),
$$

where $\bar{H}_{\tau}$ is an endomorphism of $\pi_{q}\left(G ; \Sigma^{2} A\right)$ and $\sigma_{\tau}(N)$ is an integer( $\left.{ }^{2}\right)$. For a fixed $q$ the sum on the right side contains only a finite number $c=c(q)$ of possibly nontrivial terms. It is easily seen that $\sigma_{\tau}(N)$ is a rational polynomial in $N$ without constant term. Let $d$ be the least common multiple of the denominators of the $c$ polynomials $\sigma_{\tau}$. For any $m$, obviously $m$ divides $\sigma_{\tau}(d m)$, say $\sigma_{\tau}(d m)=m \cdot \rho_{\tau}$. Now let $N=d s$, where $s$ is the given integer. Then

$$
\begin{aligned}
\mathbf{N}_{*} x & =d s x+\Sigma_{\tau} \sigma_{\tau}(d s) \bar{H}_{\tau}(x) \\
& =s\left[d x+\Sigma_{\tau} \rho_{\tau} \bar{H}_{\tau}(x)\right] .
\end{aligned}
$$

Hence $\mathrm{N}_{*} \pi_{q}\left(G ; \Sigma^{2} A\right) \subset s \cdot \pi_{q}\left(G ; \Sigma^{2} A\right)$ as asserted.

Now we turn to the proof that (3) implies (4). We consider a homology decomposition of the given map $\phi: L \rightarrow X$ (see [10]). This consists of the following sequence of spaces and maps

$$
L=L_{0} \stackrel{j_{1}}{\longrightarrow} L_{1} \stackrel{j_{2}}{\longrightarrow} \cdots \stackrel{j_{R}}{\longrightarrow} L_{R} \stackrel{\phi_{R}}{\longrightarrow} X,
$$

where $L_{r}=C K^{\prime}\left(H_{n_{r}}(\phi), n_{r}-1\right) \cup_{u_{r}} L_{r-1}$ and $\phi_{R} j_{R} \cdots j_{2} j_{1}=\phi\left({ }^{3}\right)$. Since $H_{i}(X)=0$ for $i$ sufficiently large, we choose $R$ so that $\phi_{R}$ is a homotopy equivalence. In

(2) Note that $\bar{H}_{\tau}=\bar{u}_{\tau} \circ H_{\tau}$ is the composition of a generalized Hopf invariant $H_{\tau}$ with a generalized Whitehead product $\bar{u}_{\tau}$.

(3) We denote the $i$ th homology group of the map $\phi$ by $H_{i}(\phi)$. 
order to construct the desired weak $\mathscr{F}$-equivalence $\psi: X \rightarrow L$, it suffices to construct a weak $\mathscr{F}$-equivalence $\lambda_{R}: L_{R} \rightarrow L$. This latter map is defined by induction over the $L_{r}$. For $r=0$ take $\lambda_{0}=\mathrm{Id}: L \rightarrow L$. Now let us assume that there exists $\lambda_{r-1}: L_{r-1} \rightarrow L, r-1<R$, such that, for some integer $N>0$,

$$
\lambda_{r-1} j_{r-1} \cdots j_{1}=\mathbf{N}=\mathrm{Id}+\cdots+\mathrm{Id} \text { on } L=S^{n_{1}} \vee \cdots \vee S^{n_{k}} \text {. }
$$

Consider the element $\lambda_{r-1} u_{r} \in \pi_{n_{r}-1}\left(H_{n_{r}}(\phi) ; L\right)$. Since $\phi$ is a weak $\mathscr{F}$-equivalence by hypothesis, $H_{i}(\phi)$ is a finite abelian group for all $i$. Consequently $\pi_{n_{r}-1}\left(H_{n_{r}}(\phi) ; L\right)$ is a finite abelian group of order $t$. We now apply Proposition 3.1 with $s=t$ to obtain an integer $M>0$ such that $\mathbf{M}_{*}\left(\lambda_{r-1} u_{r}\right)=0$. Hence $\mathbf{M} \lambda_{r-1}$ can be extended to a map $\lambda_{r}: L_{r} \rightarrow L$, and clearly $\lambda_{r} j_{r} \cdots j_{1}=\mathbf{P}$ for some $P=M N$. In this way we obtain $\lambda_{R}: L_{R} \rightarrow L$ such that $\lambda_{R} j_{R} \cdots j_{1}=\mathbf{S}$ for some integer $S>0$. It follows from the commutativity of the left triangle in the diagram

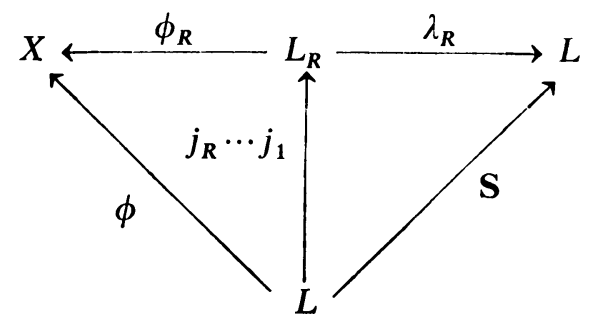

that $j_{R} \cdots j_{1}$ is a weak $\mathscr{F}$-equivalence. Therefore $\lambda_{R}$ is a weak $\mathscr{F}$-equivalence, and the proof of Theorem 2.5 is complete.

4. The kernel of the Hurewicz homomorphism. This section is dual to $\$ 2$. For this reason the proofs will often be only sketched. Here we shall deal with a Postnikov decomposition $\left\{X^{n} ; k^{n}\right\}$ of a space $X[10 ; 14]$. The polyhedron $X^{n}$ is the nth (homotopy) section of the decomposition (and not an $n$-skeleton of $X$ ). In our notation $k^{n}$, the nth $k$-invariant of $\left\{X^{n} ; k^{n}\right\}$, is an element of $H^{n+1}\left(X^{n-1} ; \pi_{n}(X)\right)$. We denote the fibre maps by $p^{n}: X^{n} \rightarrow X^{n-1}$ and the $n$-equivalences by $g^{n}: X \rightarrow X^{n}$.

PROPOSITION 4.1. The following three assertions are equivalent:

(1) The Hurewicz homomorphism $h_{n}: \pi_{n}(X) \rightarrow H_{n}(X)$ is an $\mathscr{F}$-monomorphism.

(2) For any Postnikov decomposition $\left\{X^{n} ; k^{n}\right\}$ of $X$ the nth $k$-invariant $k^{n}$ is an element of finite order in $H^{n+1}\left(X^{n-1} ; \pi_{n}(X)\right)$.

(3)There exists a map $\lambda$ from $X$ into a product of Eilenberg-MacLane spaces, $\lambda: X \rightarrow K(Z, n) \times \cdots \times K(Z, n)$, such that $\lambda_{*}: \pi_{n}(X) \rightarrow \pi_{n}(K(Z, n) \times \cdots \times K(Z, n))$ is an $\mathscr{F}$-isomorphism.

Proof. First we outline the proof of the implication $(1) \Rightarrow(2)$. Let $\Omega K=\Omega K\left(\pi_{n}(X), n+1\right)=K\left(\pi_{n}(X), n\right)$ be the fibre of $p^{n}$ and $i^{n}: \Omega K \rightarrow X^{n}$ the inclusion. It is easily seen that $i_{*}^{n}: H_{n}(\Omega K ; Q) \rightarrow H_{n}\left(X^{n} ; Q\right)$ is a monomorphism if 
$h_{n}: \pi_{n}(X) \rightarrow H_{n}(X)$ is an $\mathscr{F}$-monomorphism, where $Q$ is the group of rationals. The Serre theorem $[12$, p. 284$]$ yields the following diagram with exact row:

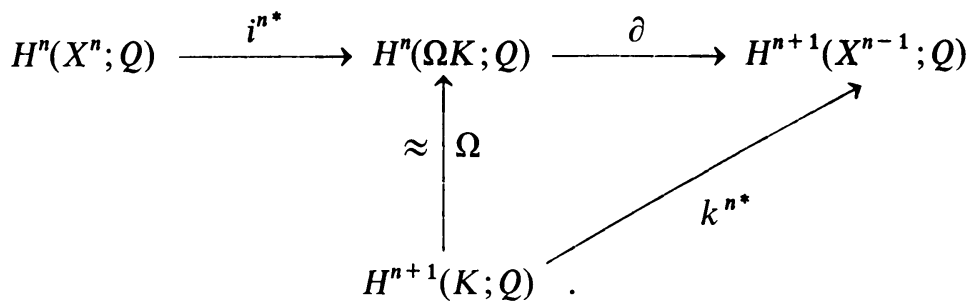

Consequently $k^{n^{*}}=0$ because $i^{n^{*}}$ is an epimorphism. This implies that $k^{n}$ is of finite order.

In order to prove $(2) \Rightarrow(3)$ we first mention the following general fact. Let $E_{\boldsymbol{f}}$ be the fiber space over $A$ induced by a map $f: A \rightarrow \Omega B$ from the Serre path-space fibration $\Omega^{2} B \rightarrow E \Omega B \rightarrow \Omega B$. Then there exists a weak $\mathscr{F}$-equivalence $\rho: E_{f} \rightarrow E_{m f}$ for any integer $m$. By taking $A=X^{n-1}, B=K\left(\pi_{n}(X), n+2\right), f=k^{n}$ and $m$ the order of $k^{n}$ we obtain a weak $\mathscr{F}$-equivalence $\rho: X^{n} \rightarrow X^{n-1} \times K\left(\pi_{n}(X), n\right)$. Then the composition

$$
X \stackrel{g^{n}}{\longrightarrow} X \stackrel{\rho}{\longrightarrow} X^{n-1} \times K\left(\pi_{n}(X), n\right) \stackrel{p}{\longrightarrow} K(Z, n) \times \cdots \times K(Z, n),
$$

where $p$ is the projection onto the $K(Z, n)$ 's appearing in $K\left(\pi_{n}(X), n\right)$, is the desired $\operatorname{map} \lambda$.

The proof that $(3) \Rightarrow(1)$ is trivial and hence omitted.

We note that Meyer [14] proves a result similar to Proposition 4.1 for the trivial class of groups.

REMARK 4.2. If $X$ is an $(r-1)$ connected space then $h_{n}: \pi_{n}(X) \rightarrow H_{n}(X)$ is an $\mathscr{F}$-monomorphism for $n \leqq 2 r-2$. To establish this known fact it is possible to dualize the proof in Remark 2.2, but we prefer to argue by means of the following diagram

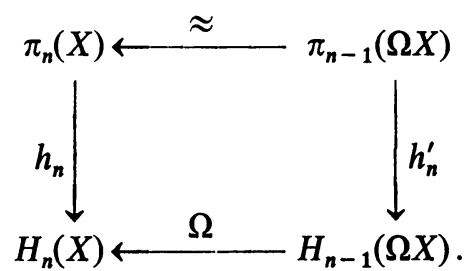

Now $\Omega$ is an isomorphism if $n \leqq 2 r-2$ [15, Proposition 10, p. 483]. By a result of Cartan and Serre [6, Remarques 1 and $3 \mathrm{~b}], h_{n}^{\prime}$ is an $\mathscr{F}$-monomorphism for all $n$. Thus $h_{n}$ is an $\mathscr{F}$-monomorphism for $n \leqq 2 r-2$.

Remark 4.3. Let $H_{n}(X)=0$ for $n>N$. Then it follows from Proposition 4.1 that for all $n>N$, the $k$-invariant $k^{n}$ is of finite order if and only if $\pi_{n}(X)$ is a finite group. 
Definitions 4.4. (a) We say that a space $X$ has finite $k$-type if in some Postnikov decomposition $\left\{X^{n} ; k^{n}\right\}$ of $X$ each $k^{n}$ is of finite order.

(b) We say that $X$ is an $H$-space mod $\mathscr{F}$ if there exists a map $\mu: X \times X \rightarrow X$ such that the composition of $\mu$ with each of the two inclusions of $X$ into $X \times X$ is a weak $\mathscr{F}$-equivalence.

The notion of an $H$-space mod $\mathscr{F}$ appears in [18]. It is dual to the notion of $\mathscr{F}$-cat $<2$.

THEOREM 4.5. Consider the following assertions:

(1) The Hurewicz homomorphism $h_{n}: \pi_{n}(X) \rightarrow H_{n}(X)$ is an $\mathscr{F}$-monomorphism for all $n$.

(2) The space $X$ has finite k-type.

(3) There exists a weak $\mathscr{F}$-equivalence

$$
\phi: X \rightarrow K\left(Z, n_{1}\right) \times \cdots \times K\left(Z, n_{k}\right) \quad\left(n_{1} \leqq n_{2} \leqq \cdots \leqq n_{k}\right) .
$$

(4) There exists a weak $\mathscr{F}$-equivalence

$$
\psi: K\left(Z, n_{1}\right) \times \cdots \times K\left(Z, n_{k}\right) \rightarrow X .
$$

(5) $X$ is an $H$-space mod $\mathscr{F}$.

Then the following conclusions hold:

(i) $(1) \Leftrightarrow(2)$.

(ii) If $\pi_{i}(X)$ is finite for all $i$ sufficiently large or if $H_{i}(X)$ is finite for all $i$ sufficiently large, then

$$
(1) \Leftrightarrow(2) \Leftrightarrow(3) \text {. }
$$

(iii) If $\pi_{i}(X)=0$ for all $i$ sufficiently large, then

$$
(1) \Leftrightarrow(2) \Leftrightarrow(3) \Leftrightarrow(4) \Leftrightarrow(5) \text {. }
$$

(iv) If $H_{i}(X)=0$ for all $i$ sufficiently large (e.g., $X$ is a finite polyhedron), then

$$
(1) \Leftrightarrow(2) \Leftrightarrow(3) \Leftrightarrow(5) \text {. }
$$

The proof of Theorem 4.5 is found in $\S 5$. The implication $(5) \Rightarrow(2)$ is a result of Thom [18, p. 36] which we establish via a theorem of Cartan-Serre (see §5). In addition, the implication (2) $\Rightarrow(5)$ constitutes a converse of Thom's theorem under the assumption that the homotopy or homology groups of $X$ vanish in sufficiently high dimensions. Example 2.7 shows that $(3) \Rightarrow(4)$ is not true if $\pi_{i}(X)$ is merely a finite group for all $i$ sufficiently large.

There is a corollary dual to Corollary 2.6 which we do not state.

5. Proof of Theorem 4.5. We begin this section with a lemma which is needed to prove (3) $\Rightarrow(4)$ in Theorem 4.5. Since a dual of the Milnor-Hilton Theorem is not available, this lemma is not as general as its corresponding assertion in $\S 3$. However, it is sufficient for our purposes. 
Lemma 5.1. Let $A=K\left(\pi_{1}, n_{1}\right) \times \cdots \times K\left(\pi_{k}, n_{k}\right)$ and $G$ be a finitely generated abelian group. Then for any given integer $s>0$ and element $x \in H^{q}(A ; G)$, there exists an integer $N>0$ such that

$$
\mathbf{N}^{*} x=s y, \quad \text { for some } y \in H^{q}(A ; G),
$$

where $\mathbf{N}: A \rightarrow A$ represents $N$ times the identity maps in $\pi(A, A)$.

Proof. It is well known $[17$, p. 169$]$ that to every $x \in H^{q}(A ; G)$ there corresponds a unique cohomology operation $T$ of type $\left(\pi_{1}, n_{1}, \cdots, \pi_{k}, n_{k} ; G, q\right)$. Indeed $x=T\left(u_{1}, \cdots, u_{k}\right)$, where $u_{i}$ is the $i$ th basic class in $H^{n_{i}}\left(A ; \pi_{i}\right)$. For any integer $N$,

$$
\mathbf{N}^{*} x=T\left(\mathbf{N}^{*} u_{1}, \cdots, \mathbf{N}^{*} u_{k}\right)=T\left(N u_{1}, \cdots, N u_{k}\right) .
$$

Thus it suffices to show that, for a given integer $s$, there exists an $N>0$ such that

$$
T\left(N u_{1}, \cdots, N u_{k}\right)=s y,
$$

for some $y \in H^{q}(A ; G)$. It has been proved (see [17, p. 172] and [9, p. 282]) that every cohomology operation $T$ is generated by the following primitive operations: (1) addition, (2) coefficient homomorphisms, (3) Bockstein-Whitney homomorphisms, (4) Steenrod reduced $p$ th powers, (5) cup products, (6) Pontryagin $p$ th powers $P_{p}$. We note that (2), (3) and (4) are additive operations and that (5) is a biadditive operation. Thus any composition of operations (1) through (5) satisfies (5.2). We next show that for any integer $m>0$,

$$
P_{p}\left(m^{2} x\right)=m y,
$$

for some $y \in H^{p q}\left(X ; Z_{p_{r+1}}\right)$. It follows by induction from formula (1.5) of [4] that

$$
P_{p}(m x)=m P_{p}(x)+\phi\left(\tau x^{p}\right),
$$

where $\phi$ is a coefficient homomorphism and $\tau$ an integer depending on $m$. Consequently

$$
\begin{aligned}
P_{p}\left(m^{2} x\right) & =m P_{p}(m x)+\phi\left(\tau(m x)^{p}\right) \\
& =m P_{p}(m x)+m^{p} \phi\left(\tau x^{p}\right) \\
& =m\left[P_{p}(m x)+m^{p-1} \phi\left(\tau x^{p}\right)\right] .
\end{aligned}
$$

This establishes (5.3). Since any cohomology operation $T$ is generated by the primitive operations (1) through (6), it follows that (5.2) holds for any T. This completes the proof of Lemma 5.1.

We now proceed to prove Theorem 4.5.

(i) By Proposition $4.1(1) \Leftrightarrow$ (2).

(ii) If $\pi_{i}(X)$ is finite for large $i$, then (1) $\Leftrightarrow(2) \Leftrightarrow(3)$ by Proposition 4.1. If $H_{i}(X)$ is finite for large $i$, then (1) implies that $\pi_{i}(X)$ is finite for large $i$. This completes the proof of (ii). 
(iii) As in the dual case, (3) and (4) imply (5). Moreover, it is easily verified that $(4) \Rightarrow(1)$. The proposition $(5) \Rightarrow(1)$ follows from a theorem of Cartan-Serre [6, Remarques 1 and $3 \mathrm{~b}]$. Thus in order to establish (iii), it remains to show that (3) $\Rightarrow(4)$ if $\pi_{i}(X)=0$ for large $i$. The proof of this is dual to the proof of (3) $\Rightarrow(4)$ in §3. One argues by means of a homotopy decomposition [10] of the map $\phi: X \rightarrow K\left(Z, n_{1}\right) \times \cdots \times K\left(Z, n_{k}\right)$, with Lemma 5.1 taking the place of Proposition 3.1 .

(iv) We have only to show that (3) $\Rightarrow(5)$, if $H_{i}(X)=0$ for all $i$ sufficiently large. Let $\left\{X^{n} ; k^{n}\right\}$ be a Postnikov decomposition of $X$ with $n$-equivalences $g^{n}: X \rightarrow X^{n}$. Clearly there exists an integer $M$ such that $H_{i}(X \times X)=0$ for $i \geqq M$, and there exists a map $\phi^{\prime}: X^{M} \rightarrow K\left(Z, n_{1}\right) \times \cdots \times K\left(Z, n_{k}\right)$ such that

$$
\phi=\phi^{\prime} g^{M}: X \rightarrow K\left(Z, n_{1}\right) \times \cdots \times K\left(Z, n_{k}\right) .
$$

Since $\phi$ is a weak $\mathscr{F}$-equivalence, so is $\phi^{\prime}$. By applying (iii) above to $X^{M}$, we conclude that $X^{M}$ is an $H$-space mod $\mathscr{F}$ with "multiplication"

$$
\mu^{M}: X^{M} \times X^{M} \rightarrow X^{M} .
$$

We consider the diagram

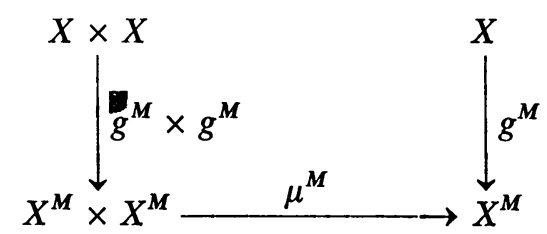

and can assume that $g^{M}$ is a fibre map with $M$-connected fibre $F$. The obstructions to a lifting of $\mu^{M}\left(g^{M} \times g^{M}\right)$ into $X$ lie in $H^{i+1}\left(X \times X ; \pi_{i}(F)\right)$. By our choice of $M$, this latter group is always trivial. Hence there exists a map $\mu: X \times X \rightarrow X$ which converts $X$ into an $H$-space mod $\mathscr{F}$.

6. Conditions under which the Hurewicz homomorphism is an $\mathscr{F}$-isomorphism.

We first recall a fact about the homotopy groups of a wedge of spheres. Let $L=S^{n_{1}} \vee \cdots \vee S^{n_{k}}$ where $n_{i} \geqq 2$ and $k>1$. Then there are certain elements $p_{1}, \cdots, p_{s}, \cdots$, called basic (Whitehead) products, in the homotopy groups of $L[11]$. If $p_{s} \in \pi_{i_{s}}(L)$ then we call $i_{s}$ the dimension of $p_{s}, i_{s}=\operatorname{dim} p_{s}$.

DEFINITION 6.1. Given a sequence of integers $\left(n_{1}, \cdots, n_{k}\right)$ with $n_{i} \geqq 2$.

Case I $(k>1)$. Let $p_{1}, \cdots, p_{s}, \cdots$ be the basic products in the homotopy groups of $S^{n_{1}} \vee \cdots \vee S^{n_{k}}$. We call an integer $n$ a Whitehead number with respect to $\left(n_{1}, \cdots, n_{k}\right)$ if (a) $n=2 \operatorname{dim} p_{s}-1$, where $\operatorname{dim} p_{s}$ is even, or if (b) $n=\operatorname{dim} p_{s}$, for some $s>k$.

Case II $(k=1)$. We call $n$ a Whitehead number with respect to $n_{1}$ if $n=2 n_{1}-1$, where $n_{1}$ is even.

In short, $n$ is a Whitehead number with respect to $\left(n_{1}, \cdots, n_{k}\right)$ if and only if $\pi_{n}\left(S^{n_{1}} \vee \cdots \vee S^{n_{k}}\right)$ contains a Whitehead product of infinite order. 
Proposition 6.2. Let $X$ have finite $k^{\prime}$-type and let $x_{1}, \cdots, x_{k}$ be a basis of $\sum_{i \leqq n} H_{i}(X ; Q)$. Then $h_{n}: \pi_{n}(X) \rightarrow H_{n}(X)$ is an $\mathscr{F}$-monomorphism if and only if $n$ is not a Whitehead number with respect to $\left(n_{1}, \cdots, n_{k}\right)$, where $n_{i}=\operatorname{dim} x_{i}$.

Proof. Let $\left\{X_{n} ; k_{n}^{\prime}\right\}$ be a homology decomposition of $X$. Since $k_{n+1}^{\prime}$ is of finite order, $j_{n+1 \neq}: \pi_{n}\left(X_{n}\right) \rightarrow \pi_{n}\left(X_{n+1}\right)$ is an $\mathscr{F}$-isomorphism. Clearly

$$
g_{n+1 *}: \pi_{n}\left(X_{n+1}\right) \rightarrow \pi_{n}(X)
$$

is an isomorphism, and so $g_{n *}: \pi_{n}\left(X_{n}\right) \rightarrow \pi_{n}(X)$ is an $\mathscr{F}$-isomorphism. By Theorem 2.5 there exists a weak $\mathscr{F}$-equivalence $\phi: L=S^{n_{1}} \vee \cdots \vee S^{n_{k}} \rightarrow X_{n}$. Now consider the diagram

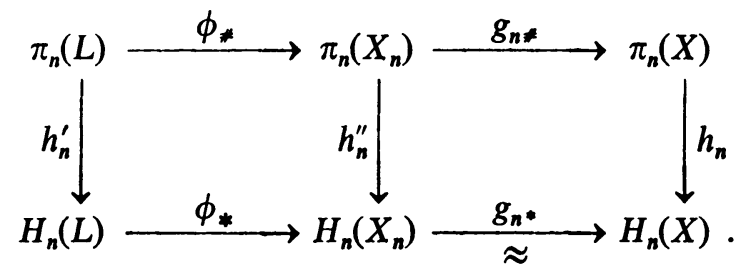

We see that $h_{n}$ is an $\mathscr{F}$-monomorphism if and only if so is $h_{n}^{\prime}$. It follows from $\left[11\right.$, Theorem A] that mod $\mathscr{F}$ the kernel of $h_{n}^{\prime}$ is generated by all Whitehead products of infinite order. Thus $h_{n}^{\prime}$ is an $\mathscr{F}$-monomorphism if and only if $n$ is not a Whitehead number with respect to $\left(n_{1}, \cdots, n_{k}\right)$.

Corollary 6.3. Let $X$ be as in Proposition 6.2. Then the nth $k$-invariant $k^{n}$ of $X$ is of infinite order if and only if $n$ is a Whitehead number with respect to $\left(n_{1}, \cdots, n_{k}\right)$, where $n_{i}=\operatorname{dim} x_{i}$. In particular, if the rational homology of $X$ has at least two generators of positive dimensions, then $k^{n}$ is of infinite order for infinitely many $n$.

We remark that one can define the notion of a cup number and prove a proposition which is dual to Proposition 6.2.

The next theorem characterizes the spaces $X$ for which $h_{n}$ is an $\mathscr{F}$-isomorphism for all $n$.

THEOREM 6.4. Assume that $H_{i}(X)$ or $\pi_{i}(X)$ is an infinite group for some $i>0$. Then $h_{n}: \pi_{n}(X) \rightarrow H_{n}(X)$ is an $\mathscr{F}$-isomorphism for all $n$ if and only if $X$ is a rational homology sphere of odd dimension (i.e., the rational homology groups of $X$ are the same as those of $S^{r}, r$ odd).

Proof. Let $X$ be a rational homology sphere of odd dimension $r$. Since all homology groups of $X$ are finite with the exception of $H_{r}(X)$, it follows that $X$ has finite $k^{\prime}$-type. Therefore $h_{n}$ is an $\mathscr{F}$-epimorphism for all $n$. Since $r$ is odd there are no Whitehead numbers relative to $r$. Hence, by Proposition $6.2, h_{n}$ is an $\mathscr{F}$-monomorphism for all $n$.

If on the other hand $h_{n}$ is an $\mathscr{F}$-isomorphism, then $X$ has finite $k^{\prime}$-type and finite $k$-type. By Corollary 6.3, the rational homology of $X$ has one generator 
in $H_{r}(X ; Q)$. In view of Proposition $6.2, r$ must be odd. Thus $X$ is a rational homology sphere of odd dimension.

COROLlaRY. 6.5. Let $H_{i}(X)$ or $\pi_{i}(X)$ be an infinite group for some $i>0$, and assume that $H_{r}(X)=0$ for all $r$ sufficiently large. Then $X$ is both an $H$-space $\bmod \mathscr{F}$ and of $\mathscr{F}$-cat $<2$ if and only if $X$ is a rational homology sphere of odd dimension.

For the proof see Theorems 2.5 and 4.5.

\section{REFERENCES}

1. M. G. Barratt, Spaces of finite characteristic, Quart. J. Math. Oxford Ser. (2) 11 (1960), 124-136.

2. I. Berstein, Homotopy mod. C of spaces of category 2, Comment. Math. Helv. 35 (1961). 9-14.

3. A. L. Blakers and W. S. Massey, The homotopy groups of a triad. II, Ann. of Math. (2) 55 (1952), 192-201.

4. W. Browder and E. Thomas, Axioms for the generalized Pontryagin cohomology operations, Quart. J. Math. Oxford Ser. (2) 13 (1962), 55-60.

5. E. H. Brown and A. H. Copeland, An homology analogue of Postnikov systems, Michigan Math. J. 6 (1959), 313-330.

6. H. Cartan and J.-P. Serre, Espaces fibrés et groupes d'homotopie. II. Applications, C. R. Acad. Sci. Paris 234 (1952), 393-395.

7. C. R. Curjel, On the homology decomposition of polyhedra, Illinois J. Math. 7 (1963), 121-136.

8. _- A note on spaces of category $\leqq 2$, Math. Z. 80 (1963), 293-299.

9. A. Dold, Ueber die Steenrodschen Kohomologie-operationen, Ann. of Math. (2) 73 (1961), 258-294.

10. B. Eckmann and P. J. Hilton, On the homology and homotopy decomposition of continuous maps, Proc. Nat. Acad. Sci. U.S.A. 45 (1959), 372-375.

11. P. J. Hilton, On the homotopy groups of the union of spheres, J. London Math. Soc. 30 (1955), 154-172.

12. S. T. Hu, Homotopy theory, Academic Press, New York, 1959.

13. I. M. James, On the homotopy groups of spheres, Sympos. Internacional de Topologia Algebraica pp. 222-224, Univ. de Mexico, 1958.

14. J.-P. Meyer, Whitehead products and Postnikov systems, Amer. J. Math. 82 (1960), 271-280.

15. J.-P. Serre, Homologie singulière des espaces fibrés, Ann. of Math. (2) 54 (1951), 425-505.

16. - Groupes d'homotopie et classes de groupes abéliens, Ann. of Math. (2) 58 (1953), 258-294.

17. N. E. Steenrod, Cohomology operations, Sympos. Internacional de Topologia Algebraica pp. 165-185, Univ. de Mexico, 1958.

18. R. Thom, L'homologie des espaces fonctionnels, Colloque de Topologie Algèbrique, pp. 29-39, Louvain, 1956.

Princeton University,

Princeton, New Jersey

CORNELl UNIVERSITY,

ITHACA, NEW YORK 